\title{
Hsa_circ_0091074 regulates TAZ expression via microRNA-1297 in triple negative breast cancer cells
}

\author{
JIASHU HU ${ }^{1,2^{*}}$, CHANGLE JI ${ }^{1 *}$, KAIYAO HUA ${ }^{1}$, XUEHUI WANG ${ }^{1}$, \\ XIAOCHONG DENG ${ }^{1}$, JIAYI LI ${ }^{1}$, DINNY GRAHAM ${ }^{2}$ and LIN FANG ${ }^{1}$
}

${ }^{1}$ Department of Thyroid and Breast, Division of General Surgery, Shanghai Tenth People's Hospital, Tongji University School of Medicine, Shanghai 200072, P.R. China; ${ }^{2}$ Centre for Cancer Research, The Westmead Institute for Medical Research, The University of Sydney, Westmead, New South Wales 2145, Australia

Received September 4, 2019; Accepted January 31, 2020

DOI: $10.3892 /$ ijo.2020.5000

\begin{abstract}
Triple negative breast cancer (TNBC) has the highest recurrence, metastasis and mortality rate of all breast cancer subtypes, due to its typically more aggressive characteristics and lack of effective targeted treatment options. The Hippo pathway is a signaling cascade composed of a group of conserved kinases, which serves an important role in almost all cancer types. Both circular RNAs (circRNAs) and microRNAs (miRNAs) are types of non-coding RNAs, which influence cancer progression. CircRNAs have been demonstrated to serve as miRNA 'sponges', binding to miRNAs to inhibit their function. In the present study, it was revealed that circular RNA hsa_circ_0091074 binds miR-1297, and that there is an inverse association between the expression levels of the two non-coding RNAs in breast cells, indicating that hsa_circ_0091074 may serve as an endogenous 'sponge' for miR-1297. Subsequently, the potential function and mechanism underlying the involvement of miR-1297 in breast cancer was investigated via MTT, colony formation, wound healing and cell cycle assays. Increased miR-1297 expression resulted in a decrease in the protein levels of critical Hippo pathway transcriptional mediator Transcriptional coactivator with PDZ-binding motif (TAZ), which is a putative target of miR-1297. This was confirmed using dual-luciferase reporter
\end{abstract}

Correspondence to: Professor Lin Fang, Department of Thyroid and Breast, Division of General Surgery, Shanghai Tenth People's Hospital, Tongji University School of Medicine, 301 Yanchangzhong Road, Shanghai 200072, P.R. China

E-mail: fanglin2017@126.com

Dr Dinny Graham, Centre for Cancer Research, The Westmead Institute for Medical Research, The University of Sydney, 176 Hawkesbury Road, Westmead, New South Wales 2145, Australia E-mail: dinny.graham@sydney.edu.au

\section{${ }^{*}$ Contributed equally}

Key words: hsa_circ_0091074, miR-1297, triple negative breast cancer, TAZ, Hippo pathway assays, which revealed that miR-1297 targets TAZ by binding its 3'-untranslated region (3'UTR). The current results indicate that miR-1297 serves as a suppressor of breast cancer cell proliferation and invasiveness, and that this can be partially reversed by hsa_circ_0091074, suggesting that the hsa_ circ_0091074/miR-1297/TAZ/TEAD4 axis may represent a potential therapeutic target for triple negative breast cancer in the future.

\section{Introduction}

The most common cancer type worldwide in women is breast cancer, with an estimated $>2$ million new breast cancer cases diagnosed in 2018, worldwide (1). Triple negative breast cancer (TNBC) is the most aggressive and invasive subtype, and account for $\sim 20 \%$ of all breast cancer cases. TNBCs lack expression of the estrogen receptor (ER) and progesterone receptor (PR), and do not express the human epidermal growth factor receptor-2 (HER2) gene. As a result of this, there are no effective targeted therapies for patients with TNBC that are currently available as standard treatments, although some are under trial. The CDK4/6 inhibitor, Palbociclib, has been approved by the U.S. Food and Drug Administration for the treatment of metastatic breast cancer, but there is not sufficient clinical data to support its effectiveness in patients with TNBC (2). TNBC tumors are typically of a higher grade and exhibit a higher proliferative and metastatic rate compared with hormone receptor-positive cancers, resulting in a lower 5-year survival rate than that of other breast cancer subtypes (3).

Circular (circ)RNAs are a novel class of non-coding RNAs, which have often been overlooked as they were considered to be by-products of the splicing process (4). However, with sequencing technology development, the importance of circRNAs is becoming more widely accepted, with their characteristics including high abundance and tissue-specific expression $(5,6)$. According to circFunBase, there are $>7,000$ circRNAs with ascribed functions (7). A major reported function of circRNAs is the binding of microRNAs (miRNAs/miRs) to serve as endogenous 'sponge' molecule for miRNAs and RNA-binding proteins, resulting in the regulation of gene expression $(8,9)$. They are also reported to regulate other RNA levels via complementary base pairing (10). CircRNAs can 
regulate protein activity and abundance via direct interaction with target proteins $(8,11)$. Consequently, circRNAs can serve as disease biomarkers. Reflecting these broad functions, circRNAs influence various physiological and pathological processes, including tumorigenesis (12). miRNAs have been demonstrated to target a range of mRNA transcripts which regulate the proliferation, migration and apoptosis of cancer cells (13) and circRNAs may influence this function. For example, circRNA CDR1 was reported to inhibit miR-7 and regulate EGFR and IGF-1R expression in colorectal cancer, inhibiting its progression (14). In addition, blocking miR-7 via CDR1 also regulated CCNE1 and PIK3CD expression in hepatocellular carcinoma, inhibiting both proliferation and invasion.

The Hippo signaling pathway is a regulator of tissue growth during organ development and regeneration, and exhibits considerable interspecies conservation (15). Transcriptional coactivator with PDZ-binding motif (TAZ), is a key downstream effector of the Hippo signaling pathway, which serves a major role in regulating cell number and differentiation, organ growth control, stem cell function and tissue development and regeneration $(16,17)$. In TAZ-deficient mice, mammary gland branching was decreased in post-pubertal virgin animals, suggesting an association between TAZ and the number of basal and luminal cells in the mammary gland (18). It is common to see elevated expression and nuclear translocation of TAZ in breast cancer (19). Moreover, the activity of TAZ in TNBC has been reported to be significantly higher compared with all other breast cancer subtypes $(20,21)$. Increased TAZ expression is associated with a more invasive breast cancer phenotype, which promotes cell transformation and epithelial-mesenchymal transition (22). Thus, targeting TAZ may represent a potential avenue for the treatment of TNBC.

In the present study research, it was revealed via dual-luciferase reporter assays, that hsa_circ_0091074 [a circRNA arising from the $\mathrm{X}$-inactive specific transcript (XIST) genomic region] directly binds miR-1297 via its 3'UTR. Furthermore, miR-1297 targeted TAZ to suppress proliferation and migration, and regulate the cell cycle of TNBC cells.

\section{Materials and methods}

Cell culture. The human breast cancer cell lines MDA-MB-231, MCF-7 and MDA-MB-468 were purchased from the Chinese Academy of Sciences. These cells were cultured in Dulbecco's modified Eagle's medium (DMEM; Gibco; Thermo Fisher Scientific, Inc.) supplemented with $10 \%$ fetal bovine serum (FBS; Gibco; Thermo Fisher Scientific, Inc.), penicillin (100 U/ml) and streptomycin $(100 \mu \mathrm{g} / \mathrm{ml})$ (PS; Enpromise, Hangzhou, China). Human normal breast cell line MCF-10A was bought from Shanghai Zhongqiao Xinzhou Biotechnology Co., Ltd. MCF-10A cells were cultured in Mammary Epithelial Cell Medium (MEpiCm, ScienCell, Research Laboratories, Inc.). Cells were incubated at $37^{\circ} \mathrm{C}$ and supplemented with $5 \% \mathrm{CO}_{2}$. MDA-MB-231 and MCF-7 were subcultured every 2-3 days, and MDA-MB-468 and MCF-10A were subcultured every 4-5 days.

Transfection assays. Hsa-miR-1297 mimic (miR-1297; forward, 5'-UUCAAGUAAUUCAGGUG-3' and reverse, 5'-CCUGAA
UUACUUGAAUU-3'), hsa-miR-1297 inhibitor (miR-1297 in; forward, 5'-CAGUACUUUUGUGUAGUACAA-3' and reverse, 5'-GUACUACACAAAAGUACUGUU-3'), negative control mimic (NC; forward, 5'-UUCUCCGAACGUGUC ACGUTT-3' and reverse, 5'-ACGUGACACGUUGGGAGA ATT-3') and negative control inhibitor (NC in; forward, 5'-CAG UACUUUUGUGUAGUACAA-3' and reverse, 5'-GUACUA CACAAAAGUACUGTT-3') were chemically synthesized by Guangzhou RiboBio Co., Ltd. Small interfering RNA (siRNA) targeting TAZ (siRNA-TAZ; forward, 5'-GGCCAGAGAUAU UUCCUUATT-3' and reverse, 5'-UAAGGAAAUAUCUCU GGCCTT-3') was chemically synthesized by Sangon Biotech Co., Ltd. siRNA targeting hsa_circ_0091074 (si-circ; forward, 5'-AGGAUAGCUGAAUGAAAUCUUTT-3' and reverse, 5'-AAGAUUUCAUUCAGCUAUCCUTT-3') and siRNA negative control (si-NC; forward, 5'-UUCUCCGAACGUGU CACGUTT-3' and reverse, 5'-ACGUGACACGUUCGGAGA ATT-3') were chemically synthesized by Integrated Biotech Solutions. Cells were added into 6-well plates, at a density of $\sim 2.5 \times 10^{4} / \mathrm{cm}^{2}$ and cultured with DMEM, supplemented with FBS and PS. When the cell confluency reached $30-50 \%$, the culture medium was replaced with DMEM without FBS and antibiotics, and $0.8 \mu \mathrm{mol} \mathrm{miR-1297,} \mathrm{miR-1297} \mathrm{in,} \mathrm{NC,} \mathrm{NC} \mathrm{in} \mathrm{or}$ si-TAZ were added to the cells, along with $4 \mu 1$ Lipofectamine ${ }^{\circledR}$ 2000 (Invitrogen; Thermo Fisher Scientific, Inc.), according to the manufacturer's instructions. After 4-6 h of incubation, the medium was replaced by DMEM with $10 \%$ FBS and $5 \mathrm{ml}$ PS, and all the cells were incubated at $37^{\circ} \mathrm{C}$ in a cell incubator supplemented with $5 \% \mathrm{CO}_{2}$ for $48 \mathrm{~h}$, before being harvested for analysis of biological function and protein expression.

Reverse transcription-quantitative (RT-q)PCR. Total RNA was extracted from MDA-MB-231, MDA-MB-468, MCF-7 and MCF-10A cells using TRIzol ${ }^{\circledR}$ reagent (Invitrogen; Thermo Fisher Scientific, Inc.), as well as the transfected MDA-MB-231 and MDA-MB-468 cells, according to the manufacturer's protocol. cDNA was generated via reverse transcription using the PrimeScript RT-PCR kit in accordance with the manufacturer's instructions (Takara Bio, Inc.). Subsequently, qPCR was performed on a 7900HT Fast RT-PCR instrument (Applied Biosystems; Thermo Fisher Scientific, Inc.). The primer sequences used were as follows: miR-1297 forward, 5'-UUC AAGUAAUUCAGGUG-3' and reverse, 5'-CAGTGCGTG TCGTGGAGT-3'; U6 forward, 5'-CTCGCTTCGGCAGCA CA-3' and reverse, 5'-AACGCTTCACGAATTTGCGT-3'. The amplification protocol was as follows: Initial denaturation for $3 \mathrm{~min}$ at $95^{\circ} \mathrm{C}$, followed by 40 cycles of denaturation at $95^{\circ} \mathrm{C}$ for $3 \mathrm{sec}$, annealing at $65^{\circ} \mathrm{C}$ for $30 \mathrm{sec}$ and elongation at $72^{\circ} \mathrm{C}$ for $20 \mathrm{sec}$. U6 was used as the reference gene. The RT-qPCR results were analyzed with the $2^{-\Delta \Delta \mathrm{Cq}}$ method (23).

Colony formation assay. MDA-MB-231 and MDA-MB-468 cells were first cultured in six-well plates. When cell confluency reached $30-50 \%$, the cells were transfected with miR-1297, miR-1297 in, NC, NC in, si-circ or si-NC as described above. After $48 \mathrm{~h}$, the treated cells were then harvested and plated into a six-well plate at a density of 500 cells/well. The plates were incubated at $37^{\circ} \mathrm{C}$ and $5 \% \mathrm{CO}_{2}$ for 7 to 10 days, with the medium changed every three days. When the colonies were visible, the medium was removed and the plates were washed 
three times with phosphate buffered saline (PBS) and allowed to dry. The dried colonies were fixed using $95 \%$ ethanol for $15 \mathrm{~min}$ at room temperature, then dried and stained with $0.1 \%$ crystal violet solution for $15 \mathrm{~min}$ at room temperature. Finally, the colonies were washed with water three times, dried and immediately imaged. Colonies were counted using a light microscope (magnification, x20).

MTT assay. MDA-MB-231 and MDA-MB-468 cells were transfected with miR-1297, miR-1297 in, NC, NC in, si-circ or si-NC as previously described. Then the transfected cells were seeded into 96-well plates at a density of 500 cells/well. Cell viability was estimated using an MTT assay kit (Sangon Biotech Co., Ltd.) at 24, 48, 72, 96 and $120 \mathrm{~h}$, according to the manufacturer's instructions. After $4 \mathrm{~h}$ incubation in MTT reagent at $37^{\circ} \mathrm{C}$ and $5 \% \mathrm{CO}_{2}$, the medium was replaced with $150 \mu 1$ dimethyl sulfoxide at room temperature (DMSO; Sangon Biotech Co., Ltd.). The absorbance of each sample was measured at $490 \mathrm{~nm}$ using a microplate spectrophotometer (BioTek Instruments, Inc.), after 10 min of agitation on a shaking table.

Wound healing assay. MDA-MB-231 cells were stored in 6-well plates, and transfected with miR-1297, miR-1297 in, $\mathrm{NC}$ or $\mathrm{NC}$ in with a range of constructs as indicated. When the cells were $~ 90 \%$ confluent, a scratch was generated in the cell monolayer by drawing a $200 \mu \mathrm{l}$ pipette tip over the surface of each well, holding the tip perpendicular to the plate. The monolayers were washed three times with PBS and cultured with DMEM medium without FBS or PS. Wound-healing was observed under a light microscope (magnification, x20) and cells were imaged at 0,24 and $36 \mathrm{~h}$ in the same position to observe cell movement.

Cell cycle assay. In total, $1 \times 10^{6}$ MDA-MB-231 cells were collected $36 \mathrm{~h}$ after transfection with miR-1297, NC, si-TAZ or si-NC, washed and centrifuged at 1,200 $\mathrm{x}$ g for $5 \mathrm{~min}$ at room temperature. The cells were suspended with pre-cooled PBS, and fixed in $75 \%$ pre-cooled ethanol at $4^{\circ} \mathrm{C}$. After $24 \mathrm{~h}$, the cells were centrifuged at $1,200 \mathrm{xg}$ for $5 \mathrm{~min}$ at $4^{\circ} \mathrm{C}$. The supernatants were then removed and RNase $(0.1 \mathrm{~g} / \mathrm{l})$ and a total of $300 \mu \mathrm{l}(0.05 \mathrm{~g} / \mathrm{l})$ propidium iodide (PI) staining solution were added to each sample to stain nuclear DNA. The cells were incubated for $30 \mathrm{~min}$ at room temperature in the dark. Cell cycle distribution was analyzed using a FACSCanto ${ }^{\mathrm{TM}}$ II flow cytometer (BD Biosciences).

Western blotting. MDA-MB-231 and MDA-MB-468 cells were cultured and transfected with miR-1297, miR-1297 in, $\mathrm{NC}, \mathrm{NC}$ in, si-TAZ and si-NC, as described above. The cells were washed three times with $4^{\circ} \mathrm{C}$ pre-cooled PBS. Whole cell protein extracts were prepared by lysis of cells using cold RIPA buffer (100 $\mu \mathrm{l} /$ well, Beyotime Institute of Biotechnology). After incubation on ice for $30 \mathrm{~min}$, the samples were centrifuged at $12,000 \mathrm{x}$ g for $30 \mathrm{~min}$ at $4^{\circ} \mathrm{C}$. Supernatants were transferred to fresh tubes, and the concentration was measured using a bicinchoninic acid (BCA) Protein assay kit (Beyotime Institute of Biotechnology), according to the manufacturer's instruction. A total of $30 \mu \mathrm{g} /$ lane of of each of the protein samples was denatured with 6x sodium dodecyl sulfate (SDS) loading buffer (Beyotime Institute of Biotechnology) at $100^{\circ} \mathrm{C}$ for $10 \mathrm{~min}$. Protein samples were separated by electrophoresis on a $10 \%$ polyacrylamide SDS gel (Beyotime Institute of Biotechnology) and transferred onto $0.45 \mu \mathrm{m}$ nitrocellulose membranes (Beyotime Institute of Biotechnology). Following 60 min of blocking with $5 \%$ fat-free milk in double distilled $\mathrm{H}_{2} \mathrm{O}$ in room temperature, membranes were incubated with primary antibodies in antibody diluent (Beyotime Institute of Biotechnology) overnight at $4^{\circ} \mathrm{C}$. Blots were washed three times with PBST (PBS containing 0.1\% Tween 20; Sangon Biotech Co., Ltd), each time for $10 \mathrm{~min}$, and incubated for $1 \mathrm{~h}$ with the anti-rabbit or mouse horseradish peroxidase-conjugated secondary antibody, as appropriate (cat. no. sc-2357; 1:2,000; Santa Cruz Biotechnology, Inc.). After three $10 \mathrm{~min}$ washes with PBST, immunoreactive protein bands were detected using an Odyssey Scanning system (LI-COR Biosciences). The density of the bands was measured using ImageStudio5.2 (LI-COR Biosciences). The following primary antibodies and dilutions were used: Matrix metalloproteinase MMP2 (cat. no. ARG40620; 1:1,000; Arigo Biolaboratories), MMP9 (cat. no. ARG22191; 1:1,000; Arigo Biolaboratories), CDK4 (cat. no. ab137675; 1:2,000; Abcam), CDK6 (cat. no. ab151247; 1:2,000; Abcam), Cyclin D1 (cat. no. ab226977; 1:2,000; Abcam), $\beta$-actin (cat. no. sc-81178; 1:2,000, Santa Cruz Biotechnology, Inc.), TAZ (cat. no. BS71120; 1:1,000; Bioworld Technology, Inc.) and TEA domain transcription factor 4 (TEAD4 cat. no. BS70599; 1:1,000; Bioworld Technology, Inc.).

Dual-luciferase reporter assay. 293T cells (Shanghai Institute of Biochemistry and Cell Biology) were seeded in 48-well plates and incubated at $37^{\circ} \mathrm{C}$ in a cell incubator supplemented with $5 \% \mathrm{CO}_{2}$. When cell confluency reached 80\%, DMEM medium was replaced with medium without FBS or PS (250 $\mu \mathrm{l} /$ well). PmirGLO-hsa_circ_0091074-miR-1297 mutant and wild type reporter plasmids, psicheck-2/TAZ 3'-UTR mutant and wild type reporter plasmids were purchased from Integrated Biotech Solutions. 293T cells were co-transfected with pmirGLO-hsa_circ_0091074-miR-1297 mutant or wild type reporter plasmid, and miR-1297 or NC vector using Lipofectamine ${ }^{\circledR} 2000$ reagent. These reagents were added to Opti-MEM (Life Technologies, Inc.) medium according to the manufacturer's instruction. 293T cells were co-transfected with psicheck-2/TAZ 3'-UTR wild type or mutant reporter plasmids, and miR-1297 or NC using Lipofectamine ${ }^{\circledR} 2000$ reagent according to the manufacturer's instruction. After $24 \mathrm{~h}$, firefly and Renilla luciferase activities were measured using a Dual-luciferase Reporter Assay (Promega Corporation), according to the manufacturer's protocol. Firefly luciferase activity was normalized to the Renilla control, and the ratio of firefly/Renilla activity was recorded.

Database analysis. StarBase v2.0 (http://starbase.sysu.edu. cn/starbase2/mirCircRNA.php), which identifies RNA-RNA and RNA-protein interaction networks, was used to predict interactions between circRNAs and miRNAs (24). CircBase (http://www.circbase.org) was used to search for the sequence of circRNAs (25). KMplotter (https://kmplot. com/analysis/index.php? $\mathrm{p}=$ service \&cancer=breast_mirna) is a webtool that combines data from the Gene Expression 


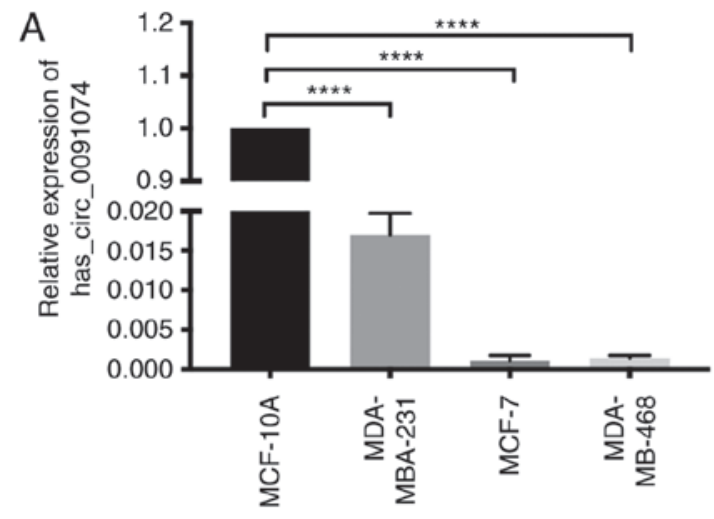

B

circRNA Name: XIST-005

circBase ID: hsa_circ_0091074

Target location: chrX:73048917-73048933

miRNA-target: miR-1297 3'- - GTG -GACTTAATGAACTT-5

-III II I:- IIIIII:

XIST-005 5'-GCACTCT-AG -CACTTGAG-3'
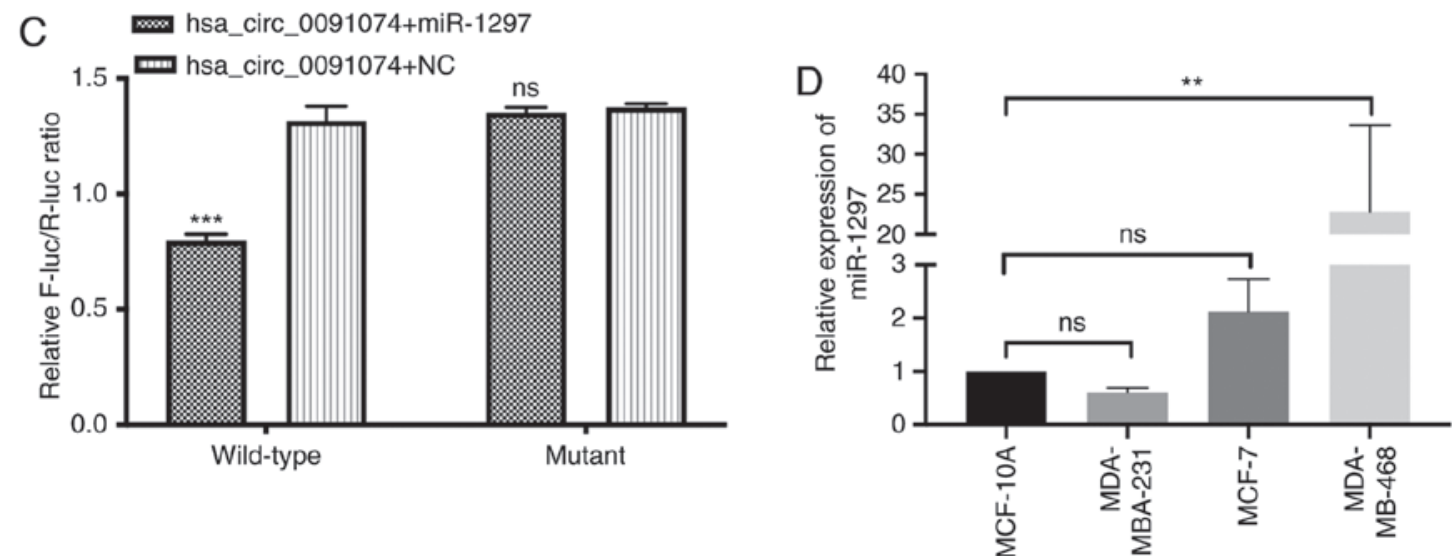

Figure 1. Expression of hsa_circ_0091074 and miR-1297 in breast cell lines, miR-1297 is a miRNA-target of hsa_circ_0091074. (A) Expression of hsa_circ_0091074 in MDA-MB-231, MCF-7 and MDA-MB-468 breast cancer cells, and MCF-10A transformed normal breast cells. One-way ANOVA. ${ }_{* * * *} \mathrm{P}<0.0001$. Dunnett's comparisons test vs. MCF-10A cells. (B) Predicted binding sites of hsa_circ_0091074 for miR-1297. (C) Dual-luciferase reporter assay evaluating the interaction between wild-type hsa_circ_0091074 + miR-1297 reporter compared with mutant hsa_circ_0091074 + miR-1297 reporter. Unpaired Student's t-test. ${ }^{* * * *} \mathrm{P}<0.001$ vs NC. (D) Expression of miR-1297 in MCF-10A, MDA-MB-231, MCF-7 and MDA-MB-468 cells. Data are presented as the mean \pm SD. One-way ANOVA and Dunnet's post-hoc test. ${ }^{* *} \mathrm{P}<0.01$ vs. MCF-10A cells. miR, microRNA; circRNA, circular RNA.

Omnibus (26), European Genome-Phenome Archive (27), The Cancer Genome Atlas (TCGA) (28) and Pubmed, and uses Kaplan-Meier survival analysis to determine the association between expression levels of potential prognostic biomarkers and clinical outcome in a range of cancers, including breast cancer (29). KMplotter was used to evaluate the association between miR-1297 or TAZ expression and breast cancer survival in TCGA datasets. TargetScan 7.2 (http://www.targetscan.org/vert_72/) was used to predict potential target genes of miRNAs (30).

Statistical analysis. Data were obtained from at least three independent experiments, and are presented as the mean \pm standard deviation (SD). Differences were considered significant for P-values $<0.05$ using the paired or unpaired Student's t-test and one-way ANOVA with Dunnett's post-hoc test. The Log-rank test was used to analyze survival in patients with breast cancer. GraphPad Prism software version 7.0 (GraphPad Software, Inc.) was used to conduct statistical analyses.

\section{Results}

Reciprocal association between hsa_circ_0091074 and miR-1297 expression in breast cancer cells. A screen of circRNAs revealed that hsa_circ_0091074 is expressed at low levels in MDA-MB-231, MCF-7 and MDA-MB-468 breast cancer cells, compared with the MCF-10A transformed normal breast cell line, which harbors a homozygous deletion of the CDKN2A tumor suppressor gene (Fig. 1A) (31). StarBase v2.0 analysis predicted that miR-1297 was a potential miRNA-target of hsa_circ_0091074, as displayed in Fig. 1B. A dual-luciferase reporter assay, using constructs containing wild-type and mutant sequences and spanning the predicted binding sites in hsa_circ_0091074, revealed a 40\% reduction in luciferase activity following transfection with the wild-type hsa_circ_0091074 + miR-1297 reporter, and no significant change with the mutant sequence-miR-1297 combination, compared with the control (Fig. 1B and C). As shown in Fig. 1D, miR-1297 is highly expressed in MCF-7 and MDA-MB-468 cells, while its expression is lower in MDA-MB-231 and MCF-10A cells. There was no significance difference between MCF-7 and MDA-MB-231 compared with MCF-10A cells. The current results indicated an inverse association between the expression levels of hsa_circ_0091074 and miR-1297. This indicates that hsa_circ_0091074 may serve as a 'sponge' RNA, targeting miR-1297.

Knockdown of hsa_circ_0091074 inhibits proliferation and viability of MDA-MB-231 cells. Hsa_circ_0091074 was knocked down in MDA-MB-231 cells and the expression of miR-1297 was measured by RT-qPCR. The knockdown of 
MDA-MB-231
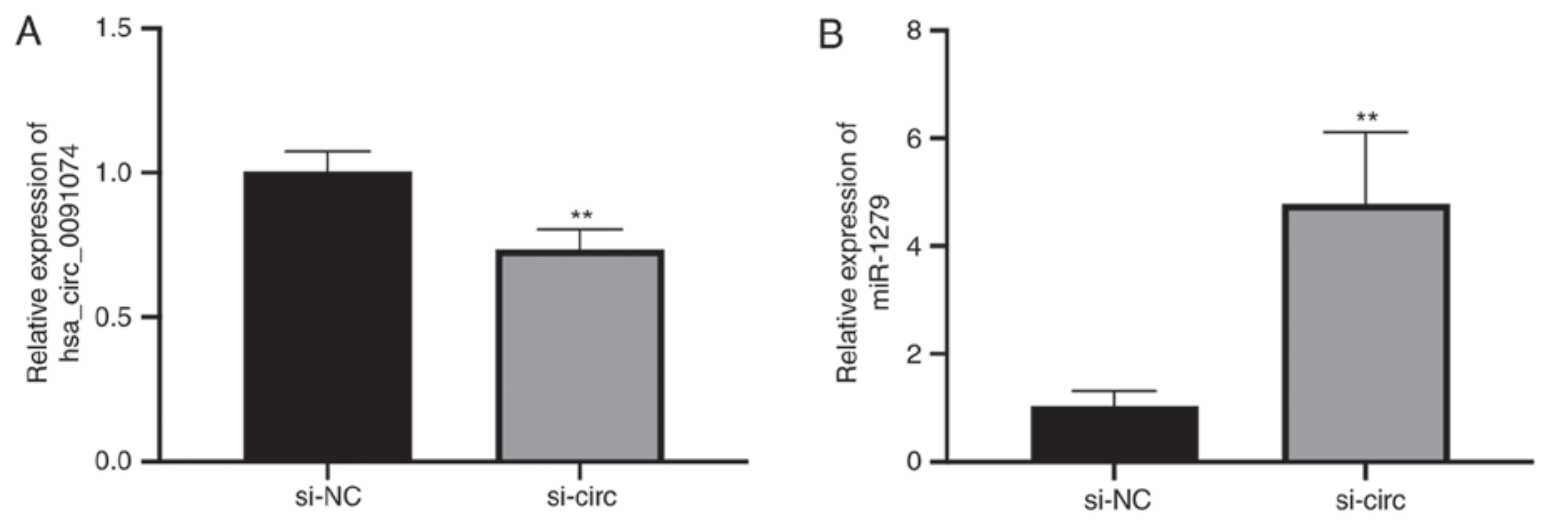

C
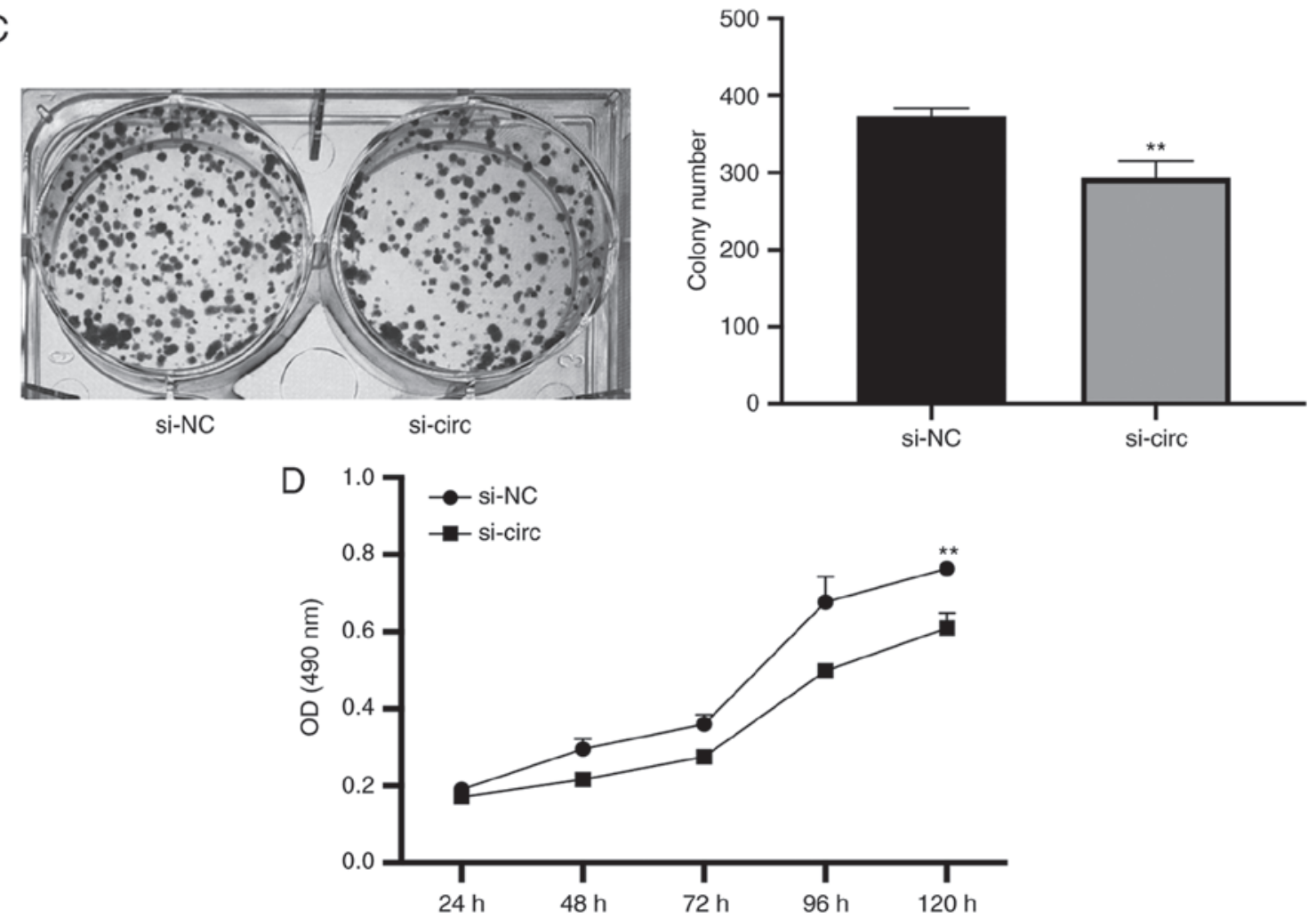

Figure 2. siRNA targeting hsa_circ_0091074 suppresses proliferation of MDA-MB-231 cells. (A) Relative hsa_circ_0091073 expression after transfection of siRNA targeting hsa_circ_0091073. Unpaired Student's t-test. " $\mathrm{P}<0.01$ vs. si-NC. (B) Relative miR-1297 expression after transfection of siRNA targeting hsa_circ_0091073. Unpaired Student's t-test. ${ }^{* *} \mathrm{P}<0.01$ vs. si-NC. (C) Effect of siRNA targeting hsa_circ_0091073 on colony formation in MDA-MB-231 cells. Data are presented as the mean \pm SD. Unpaired Student's t-test. ${ }^{* *} \mathrm{P}<0.01$ vs. si-NC. (D) Effect of siRNA targeting hsa_circ_0091073 on viability in MDA-MB-231 cells by MTT assay. Unpaired Student's t-test. ${ }^{* *} \mathrm{P}<0.01$ vs. si-NC. miR, microRNA; siRNA, small interfering RNA; NC, negative control; circRNA, circular RNA.

hsa_circ_0091074 resulted in a significant increase in the level of miR-1297 (Fig. 2A and B). Cell proliferation and viability were estimated in colony formation and MTT assays. Knockdown of hsa_circ_0091074 reduced the colony number and lowered MTT activity compared with the NC group (Fig. 2C and D). This indicates that inhibiting hsa_circ_0091074 suppresses the proliferation and viability of MDA-MB-231 cells.

miR-1297 directly inhibits the expression of TAZ. To explore the potential consequences of downregulating miR-1297 in TNBC cells, a search was performed to predict putative miR-1297 targets using the database TargetScan 7.2. Notably, although hsa_circ_0091074 (which is predicted to bind miR-1297) arises from the genomic region encoding XIST RNA, miR-1297 was not predicted to bind XIST RNA. However, the analysis predicted that TAZ was a potential target of miR-1297, and this was confirmed using a Dual-luciferase reporter assay. Constructs containing wild-type and mutant sequences spanning the predicted binding sites of miR-1297 were combined in the assay with TAZ. This revealed a highly significant $35 \%$ decrease in luciferase activity when the wild type miR-1297 construct was transfected with TAZ, whereas no change in activity was observed after transfection with the NC sequence (Fig. 3A). MDA-MB-231 and MDA-MB-468 cells were transfected with the miR-1297 mimic, NC mimic and inhibitor (in) fragments, and the expression levels of 
A

$3^{\prime}$-UTR of TAZ binding sites position (648-654):

5'...CAACATCACTTGAATCCTAG...-3' wild type 3'GUGGACUUUGAACUU5' miR-1297

5'...CAACATCTGAACTATCCTAG...3' mutant type

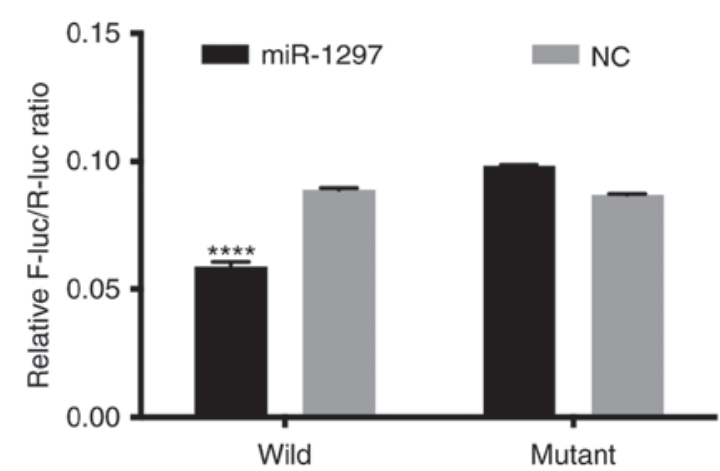

D

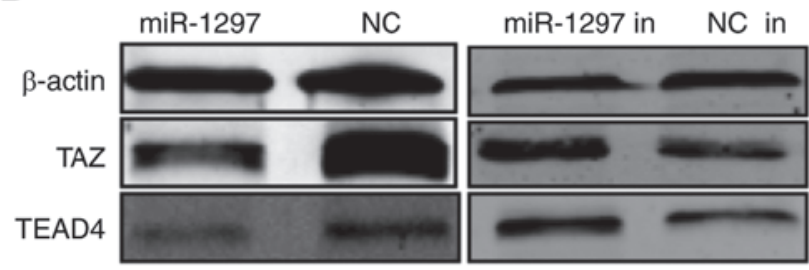

TAZ

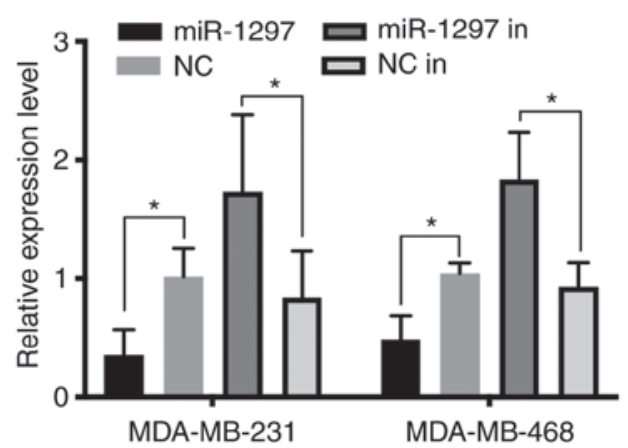

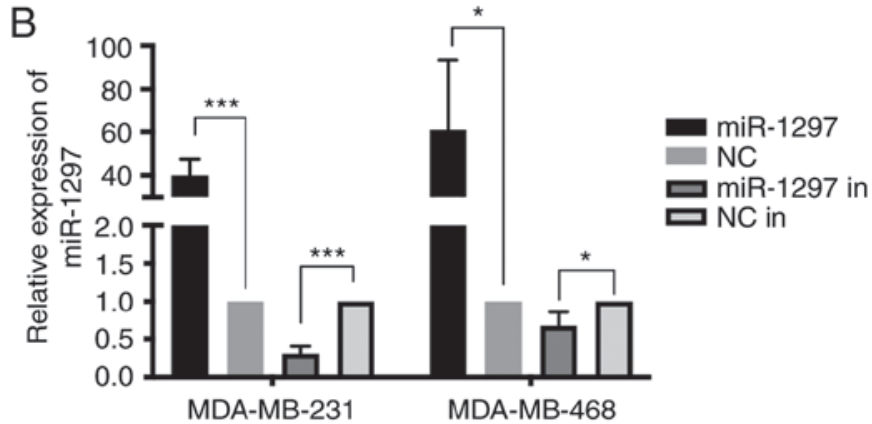

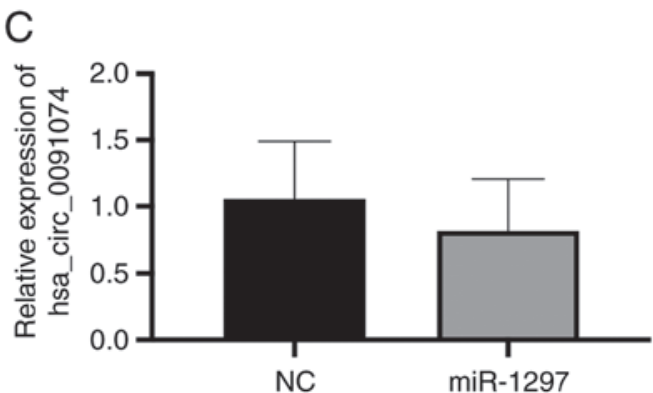

MDA-MB-468

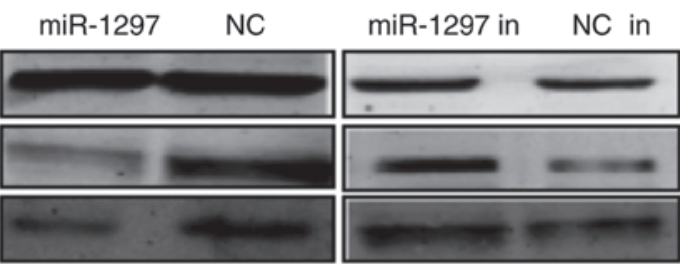

TEAD4

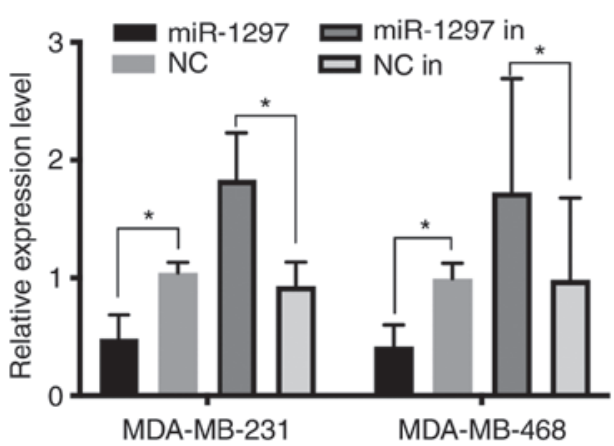

Figure 3. TAZ is a direct target gene of miR-1297. miR-1297 suppressed expression of TAZ and influenced expression of TEAD4 in TNBC cells. (A) miR-1297 binding site homology for the 3'-UTR of wild type and mutant TAZ. Dual-luciferase reporter assay for miR-1297 interaction with wild type and mutant TAZ. Unpaired Student's t-test. ${ }^{* * * *} \mathrm{P}<0.0001$ vs. NC. (B) Relative miR-1297 expression after transfection of miR-1297 mimic and miR-1297 inhibitor (miR-1297 in) in MDA-MB-231 and MDA-MB-468 breast cancer cells. Unpaired Student's t-test. ${ }^{*} \mathrm{P}<0.05,{ }^{* * *} \mathrm{P}<0.001$ vs. NC. (C) Relative hsa_circ_0091073 expression after transfection of miR-1297 mimic in MDA-MB-231 cells. Unpaired Student's t-test. (D) Impact of miR-1297 level on the expression of TAZ and TEAD4 proteins. Paired Student's t-test. Data are presented as the mean \pm SD. ${ }^{*} \mathrm{P}<0.05$ vs. NC. miR, microRNA; NC, negative control; TAZ, transcriptional coactivator with PDZ-binding motif; TEAD, TEA domain transcription factor 4.

miR-1297 were evaluated by RT-qPCR (Fig. 3B). Moreover, the expression of hsa_circ_0091074 in MDA-MB-231 was measured using RT-qPCR (Fig. 3C). After transfection with the miR-1297 mimic, the expression level of miR-1297 in the cell lines was significantly increased, and miR-1297 inhibitor markedly decreased the miR-1297 level, compared with the relevant negative controls. Western blotting analysis (Fig. 3D), revealed that overexpression of miR-1297 downregulated TAZ protein levels while transfection with the miR-1297 inhibitor resulted in a small but significant increase in TAZ protein levels, compared with the negative control inhibitor, in both MDA-MB-231 and MDA-MB-468 cells. Furthermore, miR-1297 also decreased the expression of TAZ transcriptional cofactor TEAD4, while miR-1297 inhibitor increased TEAD4 expression in MDA-MB-231 and MDA-MB-468 TNBC cells. In summary, miR-1297 downregulates TAZ expression and this influences TEAD4 expression in MDA-MB-231 and MDA-MB-468 cells. Given the association between the Hippo pathway in cancer invasion and metastasis, downregulation of components of this pathway by miR-1297 represents a potential mechanism for tumor suppression via the targeting of this molecule. 
Positive association between miR-1297 and recurrence-free survival time in $T N B C$. To investigate the potential role of miR-1297 and TAZ in TNBC, the association between miR-1297 or TAZ expression and survival was investigated in TCGA breast cancer cohort using the online tool KMplotter. The patients were grouped according to the median expression of miR-1297 or TAZ, and then the two groups were compared by Cox regression, and a Kaplan-Meier plot was drawn (29). This revealed that high expression of miR-1297 was associated with a more favorable survival time compared with the TNBC cases in which miR-1297 expression was lower (Fig. 4A). Moreover, high expression of TAZ in patients with TNBC was associated with poorer survival compared with patients with low miR-1297 expression levels (Fig. 4B). The results indicate that miR-1297 may be a tumor suppressor and that TAZ may be an oncogene in TNBC.

miR-1297 inhibits proliferation and viability of triple negative breast cancer cells. miR-1297 and miR-1297 inhibitor were overexpressed in MDA-MB-231 and MDA-MB-468 cells, and cell proliferation was estimated via colony formation and viability was estimated using MTT assays (Fig. 5A and B). miR-1297 decreased the colony number and lowered MTT activity, while transfection with miR-1297 inhibitor caused the opposite results; however, the difference of miR-1297 inhibitor in MDA-MB-231 cells was not significant. This revealed that overexpression miR-1297 inhibits cell proliferation and viability, while knockdown of miR-1297 promoted cell proliferation and viability in both cell lines. These results further support the finding that miR-1297 suppresses the proliferation and viability of TNBC cells.

miR-1297 inhibits the migration of MDA-MB-231 cells. The influence of miR-1297 on TNBC migration was tested by overexpressing either the miR-1297 mimic and miR-1297 inhibitor in MDA-MB-231 cells, and performing a wound healing assay (Fig. 6A). Cells overexpressing miR-1297 migrated markedly slower than the control group. After 24 and $36 \mathrm{~h}$, limited migration was seen in the miR-1297 overexpression group, while the wound area in the control group was almost completely filled. Although a small decrease in migration was also seen with the addition of miR-1297 inhibitor, this was not markedly different compared with the control group. Given that expression of miR-1297 in MDA-MB-231 cells is already low, it was expected that miR-1297 knockdown resulted in limited effect in these cells. Western blotting analysis (Fig. 6B), revealed that miR-1297 inhibited the expression of the migration markers MMP2 and MMP9, in MDA-MB-231 cells. These results provided further evidence that miR-1297 inhibits cell migration in MDA-MB-231 cells.

siRNA-TAZ regulates the cell cycle in MDA-MB-231 cells. To assess whether inhibition of cell proliferation by miR-1297 could be mediated by overexpressing miR-1297 or knockdown of TAZ, miR-1297, siRNA-TAZ and NC were transfected in MDA-MB-231 cells, and cell cycle distribution was analyzed by flow cytometry. The percentage of $\mathrm{G}_{0} / \mathrm{G}_{1}$ phase cells $(61.68 \%)$ increased in the si-TAZ group, compared with the control group (55.08\%). Meanwhile, the percentage of S phase cells (17.54\%) exhibited no significant difference compared
A

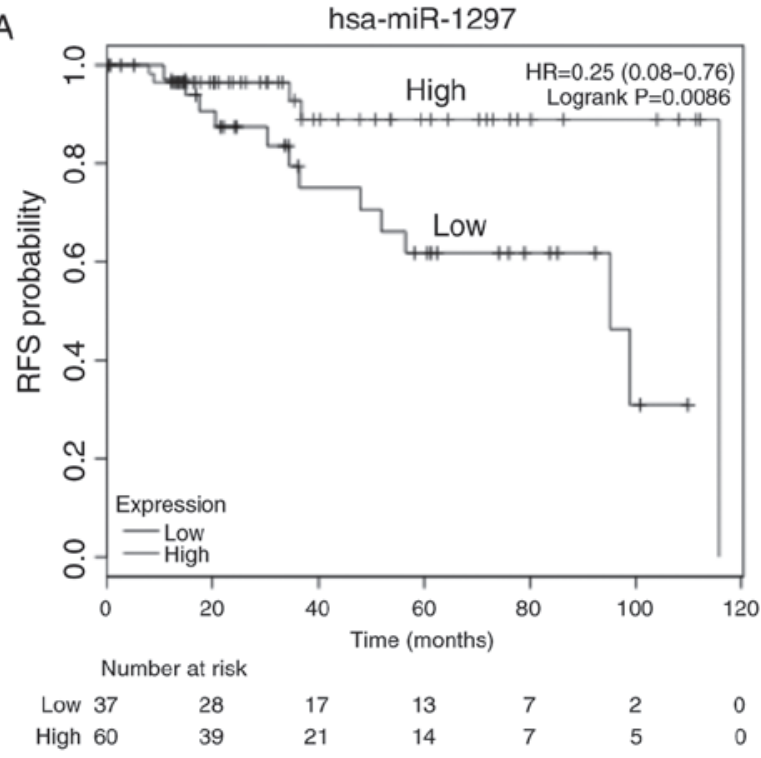

B TAZ

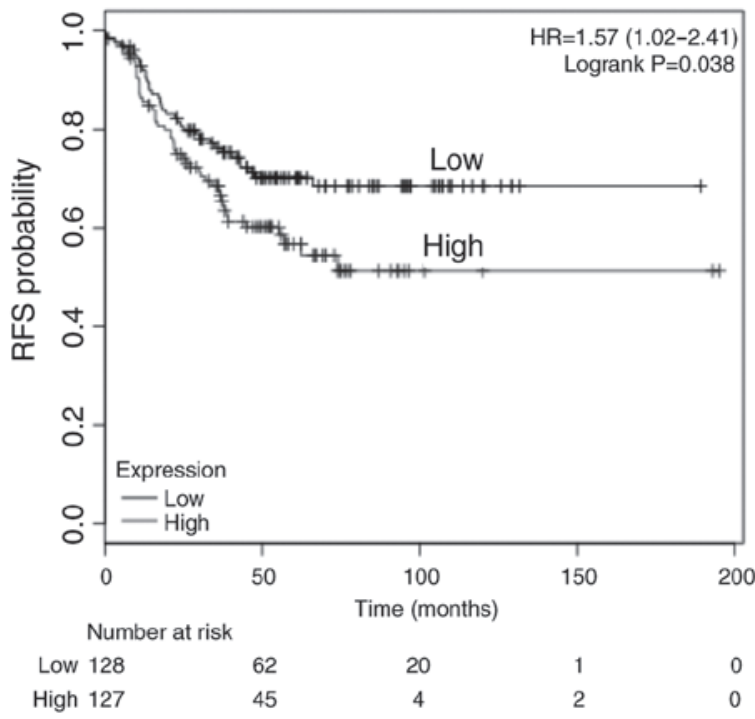

Figure 4. Expression of miR-1297 and TAZ using data from TCGA. (A) Association between miR-1297 expression and recurrence-free survival (RFS) probability in triple negative breast cancer cases from TCGA database. High expression $(n=60)$, low expression $(n=37)$. Log-rank test. $P=0.0086$ vs. low expression group. (B) Association between TAZ expression and RFS probability in TNBC cases in the TCGA database. High expression $(n=127)$, low expression $(n=128)$. Data are presented as the mean \pm SD. Log-rank test. $\mathrm{P}=0.038$ vs. low expression group. miR, microRNA; NC, negative control; TAZ, transcriptional coactivator with PDZ-binding motif; TCGA, The Cancer Genome Atlas.

with NC group (16.68\%). The percentage of $\mathrm{G}_{2} / \mathrm{M}$ phase cells decreased in the siRNA-TAZ group (20.78\%) compared with the control group (28.24\%) (Fig. 7A). Overexpressing miR-1297 also caused a $2 \%$ increase in the $G_{0} / G_{1}$ phase population compared with the control, but this difference was not statistically significant (Fig. S1). TAZ protein expression was notably reduced after transfection with siRNA-TAZ in MDA-MB-231 cells (Fig. 7B). The cell cycle markers CDK4, CDK6 and Cyclin D1 were also significantly decreased at the protein level following transfection with siRNA-TAZ. In summary, knockdown of TAZ expression regulated the 

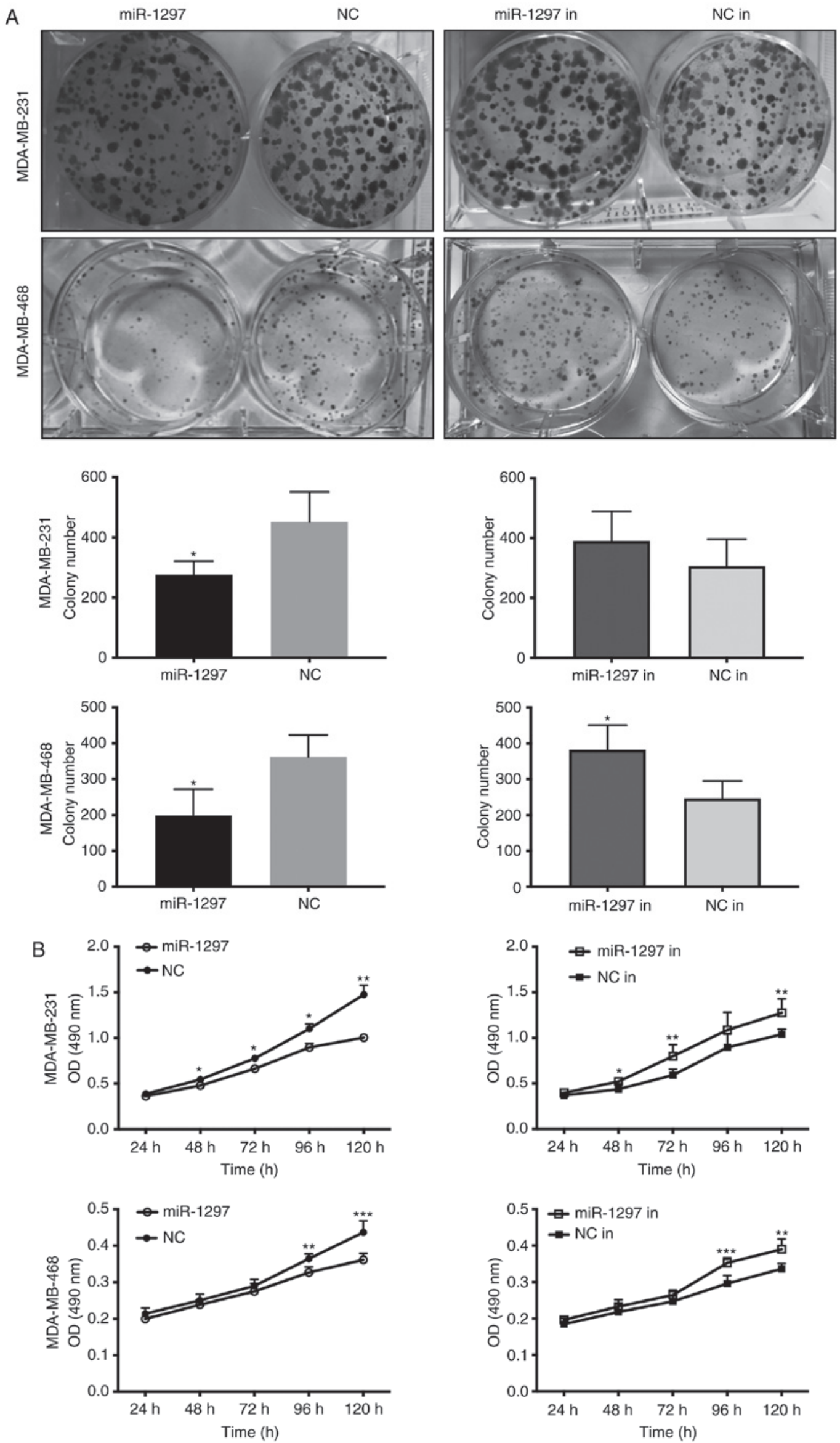

Figure 5. miR-1297 suppresses the proliferation and viability of TNBC cells. (A) Effect of miR-1297 and miR-1297 in on colony formation in MDA-MB-231 and MDA-MB-468 cells. (B) Effect of miR-1297 and miR-1297 in on viability in MDA-MB-231 and MDA-MB-468 cells by MTT assay. Data are presented as the mean $\pm \mathrm{SD}$. Unpaired Student's t-test. ${ }^{*} \mathrm{P}<0.05,{ }^{* *} \mathrm{P}<0.01,{ }^{* * *} \mathrm{P}<0.001$ vs. NC at each timepoint. miR, microRNA; NC, negative control; in, inhibitor; OD, optical density. 

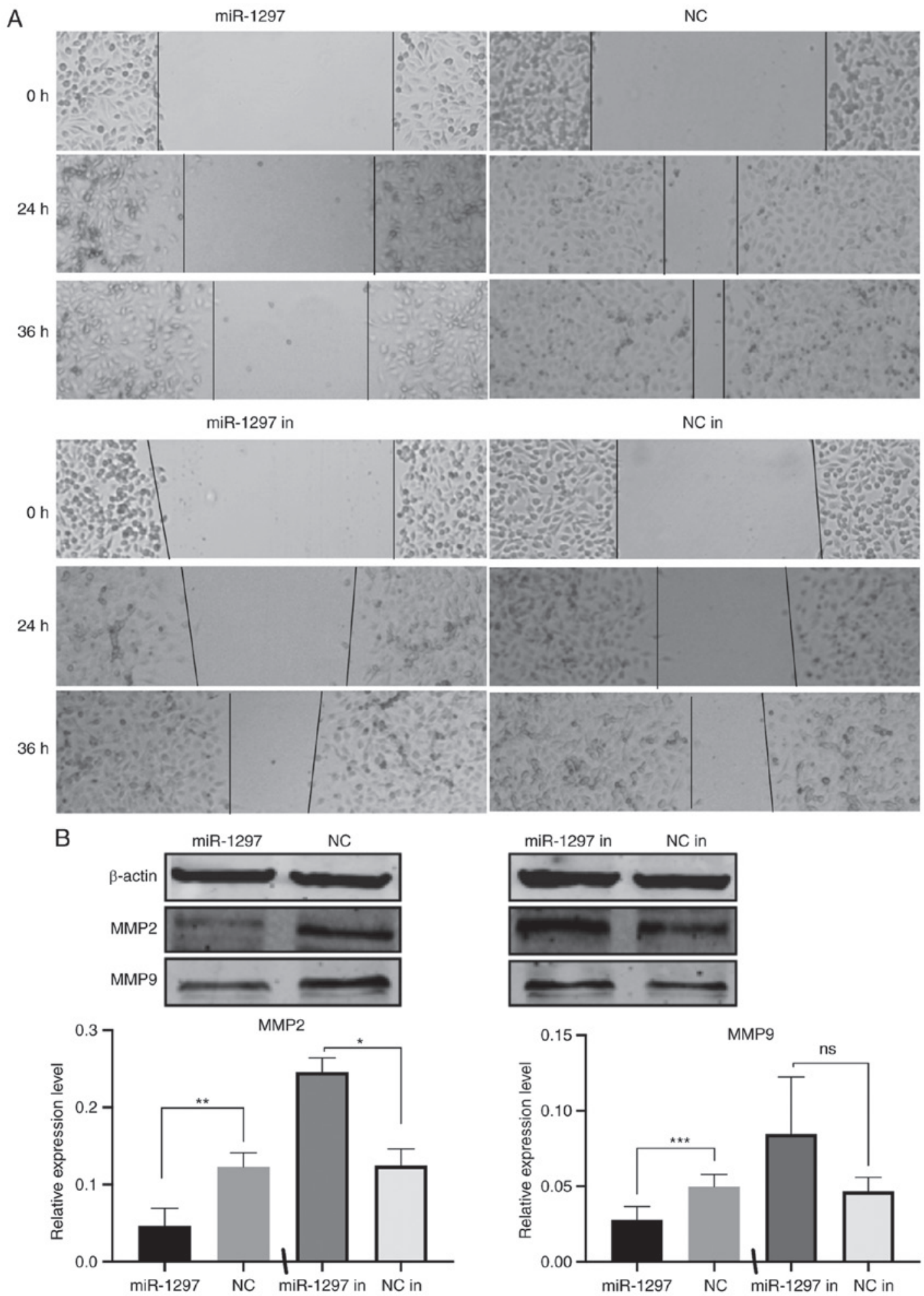

Figure 6. miR-1297 suppresses the migration of MDA-MB-231 cells. (A) Effect of miR-1297 and miR-1297 in on wound healing in MDA-MB-231 cells. (B) Effect miR-1297 and miR-1297 in on expression of MMP2 and MMP9 proteins in MDA-MB-231 cells. Paired Student's t-test. Data are presented as the mean \pm SD. ${ }^{*} \mathrm{P}<0.05,{ }^{* * *} \mathrm{P}<0.01,{ }^{* * * *} \mathrm{P}<0.001$ vs. NC. miR, microRNA; NC, negative control; in, inhibitor; MMP, matrix metalloproteinase; in, inhibitor.

$\mathrm{G}_{0} / \mathrm{G}_{1}$ and $\mathrm{G}_{2} / \mathrm{M}$ phase of MDA-MB-231 cells, consistent with a reduction in cell proliferation.

\section{Discussion}

Globally, the incidence of breast cancer has been increasing rapidly in recent years (32). TNBC is an aggressive subtype of breast cancer that exhibits high genomic heterogeneity and commonly develops resistance to existing targeted therapies (33). Consequently, when compared with other subtypes, the outcomes for patients with TNBC are often poor. For example, patients diagnosed with TNBC exhibit significantly less favorable disease-free and overall survival rates compared with those with other breast cancer types (34). 

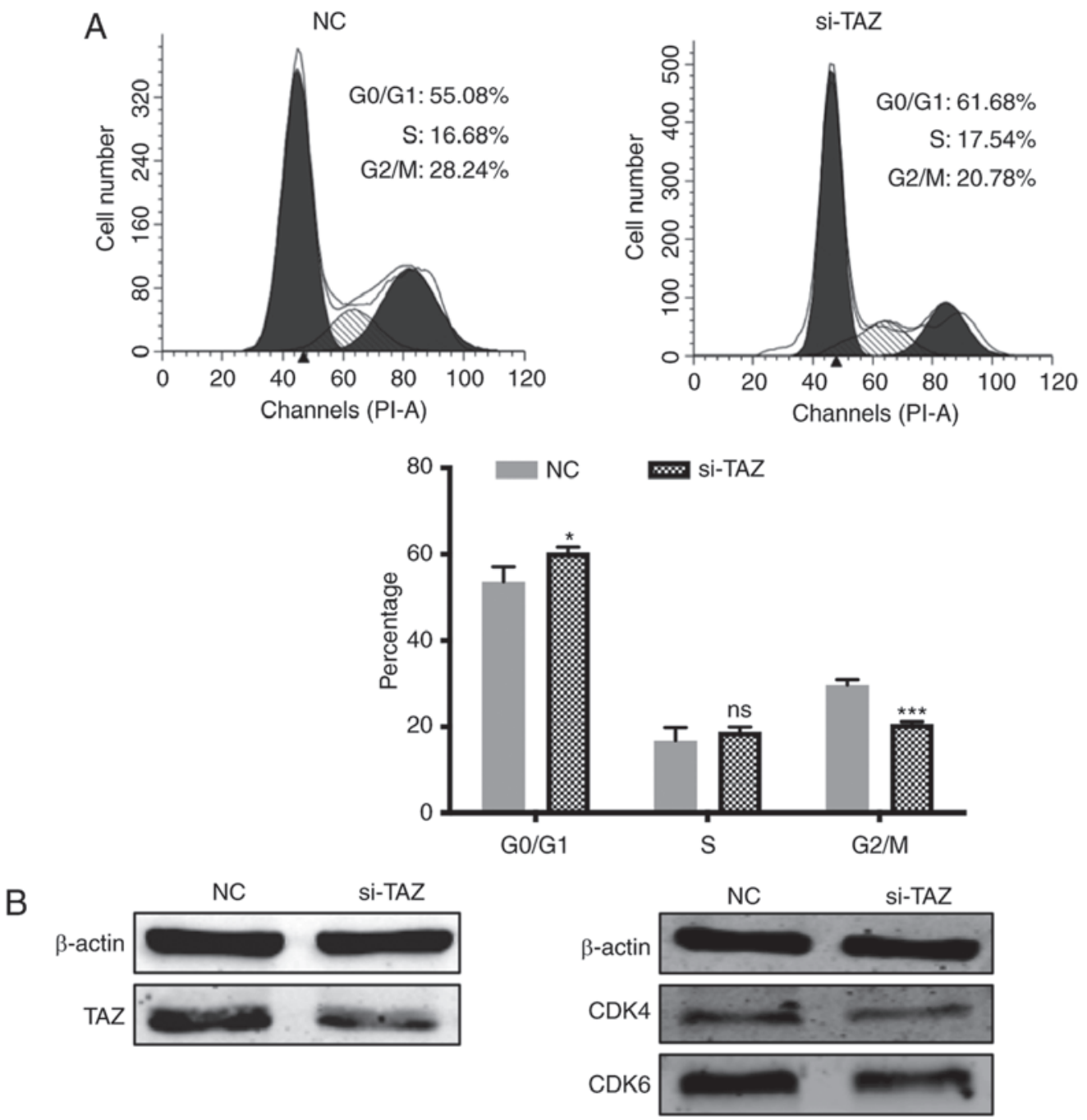

Cyclin D1
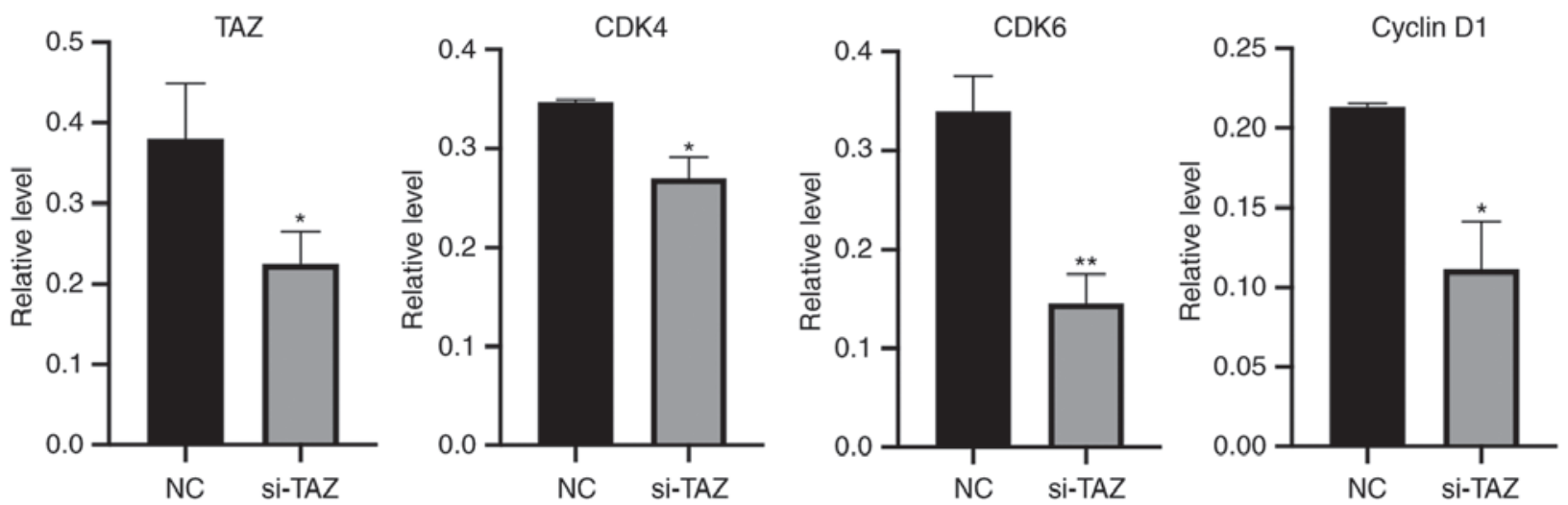

Figure 7. si-TAZ arrests MDA-MB-231 cells in the $\mathrm{G}_{0} / \mathrm{G}_{1}$ phase. (A) Effect of silencing TAZ on cell cycle distribution in MDA-MB-231 cells (distribution in $\mathrm{G}_{0} / \mathrm{G}_{1}$ : si-TAZ, $61.68 \%$; NC, 55.08\%). Unpaired Student's t-test. "P $<0.05,{ }^{* * *} \mathrm{P}<0.001$ vs. NC. (B) Impact of TAZ silencing on expression of cell cycle proteins CDK4, CDK6 and cyclin 1 in MDA-MB-231 cells. Data are presented as the mean \pm SD. Paired Student's t-test. ${ }^{*} \mathrm{P}<0.05,{ }^{* * *} \mathrm{P}<0.01$ vs. NC. miR, microRNA; NC, negative control; TAZ, transcriptional coactivator with PDZ-binding motif; siRNA, small interfering RNA.

A major function of circRNAs is the negative regulation of miRNA via binding miRNA, serving as a molecular sponge and thus decreasing the cellular abundance (35). In the present study, it was revealed that there was variable expression of hsa_circ_0091074 between different breast cell lines. It was predicted that miR-1297 was a potential target for hsa_circ_0091074. This was supported by the finding that there is an overall inverse association between the expression of hsa_circ_0091074 and miR-1297 in breast cell lines, which was most significant in the cancer cell lines, and less apparent 
in the transformed normal line. Silencing hsa_circ_0091074 resulted in an increased abundance of miR-1297, further supporting the inverse association between hsa_circ_0091074 and miR-1297. Using a Dual-luciferase reporter assay, it was confirmed that hsa_circ_0091074 binds miR-1297 by targeting its 3'UTR. Data are not currently available to compare the levels of hsa_circ_0091074 and miR-1297 in a breast cancer cohort. Therefore, it is necessary to examine whether the association between the two RNAs is observed in breast cancers, and whether there is an association with overall outcome.

MicroRNAs have gained interest due to their involvement in a plethora of different physiological processes, where they act as regulators of gene expression via post-transcriptional gene silencing. There is considerable interest in manipulating the levels of miRNAs that target tumor suppressor genes, as a potential therapeutic approach for a range of cancer types (36,37). The role of miR-1297 in cancers is controversial; miR-1297 has been reported to serve as a tumor suppressor in hepatocellular carcinoma by targeting HMGA2 (38), and in gastric cancer by targeting HMGB2 (39). The current study suggests that miR-1297 acts as a tumor suppressor in TNBC by inhibiting the oncogene TAZ. However, miR-1297 has also been reported to act as a cancer promoting factor by targeting the tumor suppressor gene PTEN in non-small cell lung cancer, laryngeal squamous cell carcinoma and testicular germ cell tumors (40-42) and in the MCF-7 and MDA-MB-453 breast cancer cell lines (43). In the present study, it was revealed that that high expression of miR-1297 was positively associated with survival in patients with TNBC using data from TCGA, and this result is consistent with a tumor suppressor role. However, further analysis revealed that higher miR-1297 was associated with a less favorable outcome in ER-positive cases in the same cohort. This analysis indicates that miR-1297 acts as a tumor suppressor in TNBC while it serves as an oncogene in ER-positive breast cancer. A range of approaches were employed to study the impact of silencing miR-1297, in both MDA-MB-231 and MDA-MB-436 cells, and the effects seen were proportional to the basal expression of miR-1297. In MDA-MB-231 cells, silencing of miR-1297 resulted in a $21 \%$ increase in proliferation, whereas in MDA-MB- 436 cells there was a $35 \%$ increase, indicating an inverse association between miR-1297 expression and cell proliferation.

The Dual-luciferase assay confirmed that the oncogene TAZ is a target gene of miR-1297, which significantly decreased luciferase activity in the wild-type group. Certain specific miRNAs simultaneously exert both oncogenic and tumor suppressive effects by silencing tumor suppressive and oncogenic mRNAs, respectively (44). Yes-associated protein (YAP) and TAZ have higher expression levels in TNBC compared with other breast cancer subtypes, providing a possible explanation for the differential association of miR-1297 with outcome in ER-positive and triple negative breast cancer (45).

As a member of the Hippo pathway, TAZ is an important oncogene in the human growth and developmental processes, with an established role as a key regulator of organ size and tissue homeostasis (46). The role of Hippo in suppressing contact inhibition and modulating cell proliferation, apoptosis and stemness, has also implicated it in cancer (47). YAP/TAZ upregulation induces cell proliferation, inflam- mation, acquisition of cancer stem cell features, metastasis formation, suppression of anoikis, reduced apoptosis and drug resistance (48). Therefore, given the high expression level of YAP/TAZ in TNBC, by targeting the Hippo pathway kinase TAZ and inhibiting its expression, miR-1297 was demonstrated to have an important function in TNBC. Although overexpression of miR-1297 did not have a significant effect on cell cycle, a small increase in $\mathrm{G}_{0} / \mathrm{G}_{1}$ fraction was observed, suggesting that miR-1297 influenced but did not significantly decrease TAZ expression. Moreover, there was a lag in time between the increase in miR-1297 expression, decrease in TAZ protein expression and the functional outcome, such that a greater effect may be observed after more sustained exposure to miR-1297. It should be noted that direct suppression of TAZ also resulted in a relatively small change in cell cycle distribution.

Taken together, the present study revealed that hsa circ_0091074 can serve as a miRNA sponge to bind miR-1297, and also aimed to elucidate the function of miR-1297 in TNBC. Via MTT, colony forming and wound healing assays, it was revealed that miR-1297 inhibited the proliferation and migration of TNBC cells. Moreover, it was demonstrated that miR-1297 directly regulates the expression of TAZ and influences the protein expression of its transcriptional cofactor TEAD4, of the Hippo pathway. The current results also indicate that miR-1297 serves as a tumor suppressor in TNBC and TAZ is an oncogene, which is upregulated in TNBC cells. CircRNA sponges targeting such miRNAs may serve as a novel method to achieve this regulation for the treatment of TNBC in the future. Future research should focus on the biological functions of circRNAs and miRNAs and their association with the Hippo pathway.

\section{Acknowledgements}

Not applicable.

\section{Funding}

The present study was supported by the Shanghai Science and Technology Commission (grant no. 17411967200) and the Shanghai Municipal Commission of Health and Family Planning (grant no. 201640097).

\section{Availability of data and materials}

The datasets analyzed during the current study are available in The Cancer Genome Atlas repository (https://www.cancer. gov/tcga).

\section{Authors' contributions}

$\mathrm{JH}$ designed, performed the experiment and wrote the manuscript. CJ co-performed the experiments and analysed data. $\mathrm{KH}$ performed western blot assays and analysed the data. XW performed RT-qPCR and analysed the data of them. $\mathrm{XD}$ acquired data by performing cell transfection, colony formation assays and counted colony numbers, then analysed and interpreted this data. JL performed the cell transfection and MTT assays, and analysed and interpreted this data. DG 
assisted in interpreting the data and was a major contributor in drafting the manuscript and revising it critically before final submission. LF helped to perform the experiment and contributed to the design of experiments. All authors read and approved the final version of the manuscript to be published.

\section{Ethics approval and consent to participate}

Not applicable.

\section{Patient consent for publication}

Not applicable.

\section{Competing interests}

The authors declare that they have no competing interests.

\section{References}

1. Ferlay J, Ervik M, Lam F, Colombet M, Mery L, Piñeros M, Znaor A, Soerjomataram I and Bray F (eds): Global Cancer Observatory: Cancer Today. IARC, Lyon, 2018. https://gco. iarc. fr/today. Accessed March 8, 2019.

2. Kwapisz D: Cyclin-dependent kinase 4/6 inhibitors in breast cancer: Palbociclib, ribociclib, and abemaciclib. Breast Cancer Res Treat 166: 41-54, 2017.

3. Woodcock CC, Huang Y, Woodcock SR, Salvatore SR, Singh B, Golin-Bisello F, Davidson NE, Neumann CA, Freeman BA and Wendell SG: Nitro-fatty acid inhibition of triple negative breast cancer cell viability, migration, invasion and tumor growth. J Biol Chem 293: 1120-1137, 2018.

4. Cocquerelle C, Mascrez B, Hétuin D and Bailleul B: Mis-splicing yields circular RNA molecules. FASEB J 7: 155-160, 1993.

5. Memczak S, Jens M, Elefsinioti A, Torti F, Krueger J, Rybak A, Maier L, Mackowiak SD, Gregersen LH, Munschauer M, et al: Circular RNAs are a large class of animal RNAs with regulatory potency. Nature 495: 333-338, 2013.

6. Qu S, Yang X, Li X, Wang J, Gao Y, Shang R, Sun W, Dou K and Li H: Circular RNA: A new star of noncoding RNAs. Cancer Lett 365: 141-148, 2015.

7. Meng X, Hu D, Zhang P, Chen Q and Chen M: CircFunBase: A database for functional circular RNAs. Database (Oxford) 2019: baz003, 2019.

8. Zhang Y, Yang L and Chen LL: Characterization of circular RNAs. Methods Mol Biol 1402: 215-227, 2016.

9. Hansen TB, Jensen TI, Clausen BH, Bramsen JB, Finsen B, Damgaard CK and Kjems J: Natural RNA circles function as efficient microRNA sponges. Nature 495: 384-388, 2013.

10. Hansen TB, Wiklund ED, Bramsen JB, Villadsen SB Statham AL, Clark SJ and Kjems J: miRNA-dependent gene silencing involving Ago2-mediated cleavage of a circular antisense RNA. EMBO J 30: 4414-4422, 2011

11. Xin Z, Ma Q, Ren S, Wang G and Li F: The understanding of circular RNAs as special triggers in carcinogenesis. Brief Funct Genomics 16: 80-86, 2017.

12. Rybak-Wolf A, Stottmeister C, Glažar P, Jens M, Pino N, Giusti S, Hanan M, Behm M, Bartok O, Ashwal-Fluss R, et al: Circular RNAs in the mammalian brain are highly abundant, conserved, and dynamically expressed. Mol Cell 58: 870-885, 2015.

13. Meng X, Zhu Y, Tao L, Zhao S and Qiu S: miR-590-3p mediates melatonin-induced cell apoptosis by targeting septin 7 in the human osteoblast cell line hFOB 1.19. Mol Med Rep 17: 7202-7208, 2018.

14. Tang W, Ji M, He G, Yang L, Niu Z, Jian M, Wei Y, Ren L and $\mathrm{Xu}$ J: Silencing CDR1as inhibits colorectal cancer progression through regulating microRNA-7. Onco Targets Ther 10: 2045-2056, 2017

15. Buglioni S, Vici P, Sergi D, Pizzuti L, Di Lauro L, Antoniani B, Sperati F, Terrenato I, Carosi M, Gamucci T, et al: Analysis of the hippo transducers TAZ and YAP in cervical cancer and its microenvironment. Oncoimmunology 5: e1160187, 2016.
16. Royer C, Koch S, Qin X, Zak J, Buti L, Dudziec E, Zhong S, Ratnayaka I, Srinivas S and Lu X: ASPP2 links the apical lateral polarity complex to the regulation of YAP activity in epithelial cells. PLos One 9: e111384, 2014.

17. Zhou X, Su J, Feng S, Wang L, Yin X, Yan J and Wang Z: Antitumor activity of curcumin is involved in down-regulation of YAP/TAZ expression in pancreatic cancer cells. Oncotarget 7: 79076-79088, 2016.

18. Shi P, Feng J and Chen C: Hippo pathway in mammary gland development and breast cancer. Acta Biochim Biophys Sin (Shanghai) 47: 53-59, 2015.

19. Plouffe SW, Hong AW and Guan KL: Disease implications of the Hippo/YAP pathway. Trends Mol Med 21: 212-222, 2015.

20. Bartucci M, Dattilo R, Moriconi C, Pagliuca A, Mottolese M, Federici G, Benedetto AD, Todaro M, Stassi G, Sperati F, et al: TAZ is required for metastatic activity and chemoresistance of breast cancer stem cells. Oncogene 34: 681-690, 2015

21. Cordenonsi M, Zanconato F, Azzolin L, Forcato M, Rosato A, Frasson C, Inui M, Montagner M, Parenti AR, Poletti A, et al: The Hippo transducer TAZ confers cancer stem cell-related traits on breast cancer cells. Cell 147: 759-772, 2011.

22. Passaniti A, Brusgard JL, Qiao Y, Sudol $M$ and Finch-Edmondson M: Roles of RUNX in Hippo pathway signaling. Adv Exp Med Biol 962: 435-448, 2017.

23. Livak KJ and Schmittgen TD: Analysis of relative gene expression data using real-time quantitative PCR and the 2(-Delta Delta C(T)) method. Methods 25: 402-408, 2001.

24. Li JH, Liu S, Zhou H, Qu LH and Yang JH: starBase v2. 0: Decoding miRNA-ceRNA, miRNA-ncRNA and protein-RNA interaction networks from large-scale CLIP-Seq data. Nucleic Acids Res 42 (Database Issue): D92-D97, 2014.

25. Glažar P, Papavasileiou P and Rajewsky N: circBase: A database for circular RNAs. RNA 20: 1666-1670, 2014.

26. Barrett T, Wilhite SE, Ledoux P, Evangelista C, Kim IF, Tomashevsky M, Marshall KA, Phillippy KH, Sherman PM, Holko M, et al: NCBI GEO: Archive for functional genomics data sets-update. Nucleic Acids Res 41 (Database Issue): D991-D995, 2013.

27. Lappalainen I, Almeida-King J, Kumanduri V, Senf A, Spalding JD, Ur-Rehman S, Saunders G, Kandasamy J, Caccamo M, Leinonen R, et al: The European Genome-phenome Archive of human data consented for biomedical research. Nat Genet 47: 692-695, 2015.

28. Tomczak K, Czerwińska P and Wiznerowicz M: The Cancer Genome Atlas (TCGA): An immeasurable source of knowledge. Contemp Oncol (Pozn) 19: A68-A77, 2015.

29. Lánczky A, Nagy Á, Bottai G, Munkácsy G, Szabó A, Santarpia L and Gyórffy B: miRpower: A web-tool to validate survival-associated miRNAs utilizing expression data from 2178 breast cancer patients. Breast Cancer Res Treat 160: 439-446, 2016.

30. Agarwal V, Bell GW, Nam JW and Bartel DP: Predicting effective microRNA target sites in mammalian mRNAs. Elife 4: e05005, 2015.

31. Cowell JK, LaDuca J, Rossi MR, Burkhardt T, Nowak NJ and Matsui S: Molecular characterization of the $t(3 ; 9)$ associated with immortalization in the MCF10A cell line. Cancer Genet Cytogenet 163: 23-29, 2005.

32. Bray F, Ferlay J, Soerjomataram I, Siegel RL, Torre LA and Jemal A: Global cancer statistics 2018: GLOBOCAN estimates of incidence and mortality worldwide for 36 cancers in 185 countries. CA Cancer J Clin 68: 394-424, 2018.

33. Beatty A, Fink LS, Singh T, Strigun A, Peter E, Ferrer CM, Nicolas E, Cai KQ, Moran TP, Reginato MJ, et al: Metabolite profiling reveals the glutathione biosynthetic pathway as a therapeutic target in triple-negative breast cancer. Mol Cancer Ther 17: 264-275, 2018

34. Chen LL, Zhang ZJ, Yi ZB and Li JJ: MicroRNA-211-5p suppresses tumour cell proliferation, invasion, migration and metastasis in triple-negative breast cancer by directly targeting SETBP1. Br J Cancer 117: 78-88, 2017.

35. Verduci L, Strano S, Yarden Y and Blandino G: The circ RNA-micro RNA code: Emerging implications for cancer diagnosis and treatment. Mol Oncol 13: 669-680, 2019.

36. Hosseinahli N, Aghapour M, Duijf PH and Baradaran B: Treating cancer with microRNA replacement therapy: A literature review. J Cell Physiol 233: 5574-5588, 2018.

37. Shah MY, Ferrajoli A, Sood AK, Lopez-Berestein G and Calin GA: microRNA therapeutics in cancer-an emerging concept. EBioMedicine 12: 34-42, 2016. 
38. Liu Y, Liang H and Jiang X: MiR-1297 promotes apoptosis and inhibits the proliferation and invasion of hepatocellular carcinoma cells by targeting HMGA2. Int J Mol Med 36: 1345-1352, 2015.

39. Li J, Gao J, Tian W, Li Y and Zhang J: Long non-coding RNA MALAT1 drives gastric cancer progression by regulating HMGB2 modulating the miR-1297. Cancer Cell Int 17: 44, 2017.

40. $\mathrm{Bu} \mathrm{W}$ and Luo T: miR-1297 promotes cell proliferation of non-small cell lung cancer cells: Involving in PTEN/Akt/Skp2 signaling pathway. DNA Cell Biol 36: 976-982, 2017.

41. Li X, Wang HL, Peng X, Zhou HF and Wang X: miR-1297 mediates PTEN expression and contributes to cell progression in LSCC. Biochem Biophys Res Commun 427: 254-260, 2012.

42. Yang NQ, Zhang J, Tang QY, Guo JM and Wang GM: miRNA-1297 induces cell proliferation by targeting phosphatase and tensin homolog in testicular germ cell tumor cells. Asian Pac J Cancer Prev 15: 6243-6246, 2014.

43. Liu C, Liu Z, Li X, Tang X, He J and Lu S: MicroRNA-1297 contributes to tumor growth of human breast cancer by targeting PTEN/PI3K/AKT signaling. Oncol Rep 38: 2435-2443, 2017.

44. Svoronos AA, Engelman DM and Slack FJ: OncomiR or tumor suppressor? The duplicity of microRNAs in cancer. Cancer Res 76: 3666-3670, 2016.
45. Liu J, Ye L, Li Q, Wu X, Wang B, Ouyang Y, Yuan Z, Li J and Lin C: Synaptopodin-2 suppresses metastasis of triple-negative breast cancer via inhibition of YAP/TAZ activity. J Pathol 244: 71-83, 2018

46. Yu FX, Zhao B and Guan KL: Hippo pathway in organ size control, tissue homeostasis, and cancer. Cell 163: 811-828, 2015.

47. Park JH, Shin JE and Park HW: The role of hippo pathway in cancer stem cell biology. Mol Cells 41: 83-92, 2018.

48. Ferraiuolo M, Verduci L, Blandino G and Strano S: Mutant p53 protein and the hippo transducers YAP and TAZ: A critical oncogenic node in human cancers. Int J Mol Sci 18: pii: E961, 2017. 